\title{
Competitive Influence Maximization on Online Social Networks under Cost Constraint
}

\author{
Bo-Lun Chen ${ }^{1,2}$, Yi-Yun Sheng ${ }^{1 *}$, Min Ji ${ }^{1}$, Ji-Wei Liu ${ }^{1}$, Yong-Tao Yu ${ }^{1}$, and Yue Zhang ${ }^{1}$ \\ ${ }^{1}$ College of Computer Engineering, Huaiyin Institute of Technology \\ Huaian, 223003, China \\ [e-mail : yiyun_shen@163.com,jimin@hyit.edu.cn, 2471536510@qq.com, allennessy@qq.com, \\ 298286132@qq.com] \\ ${ }^{2}$ Department of Physics, University of Fribourg, Fribourg, CH-1700, Switzerland \\ [e-mail: chenbolun1986@163.com] \\ *Corresponding author: Yi-Yun Sheng
}

Received February 5, 2021; revised March 4, 2021; March 24, 2021;

published April 30, 2021

\begin{abstract}
In online competitive social networks, each user can be influenced by different competing influencers and consequently chooses different products. But their interest may change over time and may have swings between different products. The existing influence spreading models seldom take into account the time-related shifts. This paper proposes a minimum cost influence maximization algorithm based on the competitive transition probability. In the model, we set a one-dimensional vector for each node to record the probability that the node chooses each different competing influencer. In the process of propagation, the influence maximization on Competitive Linear Threshold (IMCLT) spreading model is proposed. This model does not determine by which competing influencer the node is activated, but sets different weights for all competing influencers. In the process of spreading, we select the seed nodes according to the cost function of each node, and evaluate the final influence based on the competitive transition probability. Experiments on different datasets show that the proposed minimum cost competitive influence maximization algorithm based on IMCLT spreading model has excellent performance compared with other methods, and the computational performance of the method is also reasonable.
\end{abstract}

Keywords: Competitive Influence Maximization, Transition Probability, Cost Constraint, Social Networks 


\section{Introduction}

$\mathbf{W}_{\text {ith the explosive growth of the amount of information in social networks, the scope and }}$ speed of information dissemination are also changing with each passing day. The way people collect and spread in social networks is also more effective than ever, further accelerating interpersonal communication and information spreading. To some extent, this new information dissemination mechanism is built on the basis of interpersonal communication and mass communication, with the characteristics of distributed. [1, 2]. In this communication mechanism, the information dissemination between different users will be affected by their influence [3,4]. Therefore, it is vital to carry out influence analysis research [5].

The issue of maximizing influence is one of the crucial topics of influence analysis $[6,7]$. In 2015, Morone and Makse conducted an in-depth discussion of the issue of influence maximization in social networks on Nature [8]. The influence maximization problem is to find some nodes in the network, so that they can maximize the influence of other nodes in the network under a specific propagation model[9]. In general, the behavior and operation of users are often influenced by other users. Conversely, some of the user's own thoughts and actions will also affect others. For example, in the process of new product promotion, according to the characteristics of influence communication, enterprises often occupy market share through viral marketing in social networks [10,11]. In addition, enterprises often need to find the users who play the core role when they make publicity strategies, and maximize the effect of advertising according to their own importance. The selection of influence seed node can also provide effective help for real-time monitoring, prediction and early warning and emergency response services of disasters and terrorist attacks $[12,13]$. Therefore, we need to make good use of modern tools to carry out research on influence maximizing and improve public service.

Influence not only plays a huge role in public activities, but also is widely used in many scientific research fields and commercial studies, such as link prediction [14, 15], recommendation system [16, 17], Image Set classification [18, 19] and advertising [20]. Through research on related issues, researchers have found that individuals with greater influence are very important in the emergence and development of collective behavior, the promotion of new products, the dissemination and orientation of public opinion. Through this user-user interaction, influence can greatly reduce the cost of promoting new products and generate greater commercial value and also social influence [21]. Many companies now offer some free trials before launching new products. Customers are usually told that these free trial chances are randomly selected. In fact, those who really have these opportunities are often those with greater influence. Giving these influencers' opportunities will affects more customers at a limited cost. Therefore, the major question becomes how to select a specified user set, so that the user collections that are finally influenced are the largest to fulfill the marketing purpose.

However, with the increasing number of social network users, more and more users participate in product marketing, information spreading, etc. on social networks, which inevitably brings about the problem of competitive influence spreading. Although the issue of influence spreading in social networks has seen many research results in recent years, most of them are based on the assumption that there exists no competition between different influences given a constrained cost. Related research is still relatively small while the real-world problem 
of competitive influence under a certain cost is widespread. We can define these problems as the problem of winning the competition with the minimal cost, that is, we assume that we want to exceed the influence of all other competitors, and that the cost we need to achieve this goal is minimal. Therefore, the problem of the influence of competition is a more complicated problem. How to choose an effective strategy to carry out the influence spreading is both more meaningful and challenging than the traditional influence spreading problem.

\section{Related Works}

In recent years, many influence spreading models and algorithms to maximize the influence have been proposed, and have received the attention of many experts in different fields. The issue of influence maximizing under competitive conditions was first proposed by Bharathi, and in the subsequent researches, scholars mainly replied on some traditional influence propagation models, such as independent cascade model, and the expansion of these models to maximize the impact of competition $[22,23]$.

Based on this deterministic information dissemination model, Pham et al. proposed a new method to remedy the problem of competitive influence maximization (CIM). Their method showed that the complexity of the objective function was estimated to be $\mathrm{O}\left(n^{2}\right)$, and two effective algorithms were proposed to solve the CIM problem. Their results on four benchmark datasets showed that their algorithms were superior over other algorithms, especially in terms of runtime on medium-scale networks [24]. In addition, they proposed a Sandwich Approximation based on Polling-Based Approximation (SPBA). Their experiments on real social networks showed that their algorithms were more efficient and scalable than other recent methods. Specifically, their algorithms can scale up to a million-level network in just 1.5 minutes [25].

Yan et al. proposed the Threshold Influence (TI) problem, which was to choose the seed set with the least cost in order that the total influence reached a given threshold $\eta$. Then, they also proposed two models of influence diffusion, called one-to-many (OTM) and one-to-one (OTO) [26]. Gao et al. designed a sub-module utility function based on which they transformed the problem into a non-trivial set cover problem with nonlinear constraints. After verifying the NP hardness of Minimum Cost Seed Selection (MCSS), they proposed a greedy algorithm named G-MCSS to solve it. In addition, they extended the MCSS problem to a complex situation where the number of influences acceptable to each user was limited and the cost was proportional to this number. They further presented another greedy algorithm to solve the problem as well [27]. Wang et al. proposed a fluid diffusion influence propagation model, which revealed the time evolution of diffusion process by using the theory of fluid dynamics. In their work, they modeled the influence diffusion process as a three-dimensional fluid update process: fluid height difference, fluid temperature and temperature difference [28]. Chen et al. proposed a constrained diffusion independent cascade model for the influence maximizing of the lowest cost, which considered the heterogeneous communication ability of users in information dissemination[29]. Wang et al. proposed the influence graphical communication model to simulate the dynamic information transmission process of users, and proved that the problem of maximizing influence under cost constraints was NP complete [30].

Zhu et al. studied that in a competitive environment, how people chose seeds at the lowest 
cost so that their influence can reach the ideal threshold in the competition against competitors. They found that the spreading of specific influences was monotonic and sub-mode, and these fine properties made the performance of the algorithm easy to handle. Then, they proposed the Minimum Cost Seed Set problem (MinSeed) to study the initial problems and gave a greedy algorithm. They analyzed the ratio of greedy algorithms and gave results that were significantly better than those analyzed by others. Considering that the computation on real information spreading was difficult to calculate using simple greedy algorithm, they designed an effective method to estimate the information spreading in the Competitive - Independent Cascade (C-IC) model, and designed a scalable algorithm for large social networks [31]. Yang et al. proposed a multi-objective discrete particle swarm optimization algorithm (MODPSOIM-CM) in order to capture the characteristics of the actual network more effectively. The algorithm measured the cost and influence of each node at the same time, and used the heuristic method based on degree and turbulence operator to accelerate the convergence of the algorithm. In addition, they also designed a local strategy to obtain more non-dominated solutions. Their experiments showed that their algorithms had both effectiveness and efficiency in solving influence maximization of minimum cost (IM-CM) [32].

So far, some competitive influence spreading models have been made and the influence maximization algorithms have been designed based on these models[P5]. However, these models do not take into account that the temporal effect, that user's interest changes with time. There may be different probabilities for different users to be influenced by different products in different time periods. Therefore, in this paper, an influence maximization model based on the transition probability of competition is proposed, and we design the least significant cost optimization algorithm accordingly.

\section{Problem Formulation}

In the problem of winning competition with the minimal cost, we use directed graphs to represent online social networks. Among them, the number of nodes in the network is represented by $V$, and the number of edges is represented by $E$. Suppose we have $m$ products that are competing in the network, and we denote the probability matrix of different competitors chosen by certain nodes by $P$. The probability matrix that different users choose different competitors at time $t$ is denoted by $P^{t}$, in which $p_{i j}^{t}$ represents the probability that node $i$ chooses $j$ at time $t$.

$$
P^{t}=\left[\begin{array}{c}
P_{1}^{t} \\
P_{2}^{t} \\
\ldots \\
P_{n}^{t}
\end{array}\right]=\left[\begin{array}{c}
\left(p_{11}^{t}, p_{12}^{t}, \ldots, p_{1 m}^{t}\right) \\
\left(p_{21}^{t}, p_{22}^{t}, \ldots, p_{2 m}^{t}\right) \\
\ldots \\
\left(p_{n 1}^{t}, p_{n 2}^{t}, \ldots, p_{n m}^{t}\right)
\end{array}\right]
$$

where $\sum_{j=1}^{m} p_{i j}^{t}=1$ 


\subsection{IMCLT Model}

In the IMCLT model, at the beginning, we use $S, C_{1}, C_{2}, \ldots C_{\mathrm{m}-1}$ to denote the set of seed nodes of the existing $m$-type competitors( $S$ represents the set of the target product), and all nodes in the seed set are activated, and the other nodes are inactive. In the process of influence spreading, each node in the network can be activated by different competitors or stay inactive. If there is a directed edge between nodes $u$ and $v$, we denoted $w_{u v}$ as the weight of influence of the edge and the following conditions are met:

$$
\sum_{u \in \Gamma(v)} w_{u v} \leq 1
$$

where $\Gamma(v)$ is the node set pointing to node $v$. Each node $v$ has a threshold $\theta_{v}$ in the range of 0 to 1 representing the minimum influence required to activate $v$. If all of the activated neighbors of $v$ have an overall influence greater than or equal to the threshold, node $v$ is activated. The activation formula for node $v$ is formatted as follows:

$$
\sum_{i=1}^{m}\left(\frac{\left|C_{i}\right|}{n} \sum_{u \in \Gamma(v) \& u \in C_{i}} w_{u v}\right) \geq \theta_{v}
$$

Under this model, once the influence threshold is met, the node is activated. Therefore, the spreading process of the multi-competitive weighted linear threshold is as follows:

(1) In the initial state, i.e., $t=1$, all seed sets are active and other nodes are inactive.

(2) In step $t$, any node activated before step $t$ remains active, and any inactive node $v$, if the sum of the activated neighbors' weighted influences exceed the threshold of node $v$, then node $v$ becomes activated.

(3) Repeat the above process until no nodes in the network can be activated.

\subsection{Winning competition with the minimal cost}

We use the IMCLT model as the influence spreading model for this problem. Our purpose is to find a seed set $S$ that makes the influence of $S$ exceed the influence of any competitor $C$ and is cost-minimal. We use $S p(S)$ to represent the probability that a node is affected by $S$ in the network, and $S p(C)$ to represent the sum of the probability of nodes in the network that are affected by the largest competitor $C_{m}$. We use $\sigma(S, C)$ to represent the difference between the probability of the node affected by the set $S$ and the probability of the number of nodes affected by the largest competitor $C_{m}$. The specific formula is computed as follows:

$$
\sigma(S, C)=\operatorname{Sp}(S)-\operatorname{Sp}(C)=\sum_{i=1}^{n} P_{i s}^{t}-\sum_{i=1}^{n} P_{i m}^{t}
$$

In social networks, the cost of choosing different nodes as seed nodes is different, so we use $\operatorname{cost}(v)$ to represent the cost of node $v$. In this paper, we use the PageRank algorithm as a 
measure of node cost. We use $\operatorname{cost}(S)=\sum_{v \in S} \operatorname{cost}(v)$ to represent the total cost of the seed node, and $\operatorname{cost}\left(C_{i}\right)=\sum_{v \in C_{i}} \operatorname{cost}(v)$ is the total cost of the competitor $C_{i}$. Therefore, the goal of maximizing the influence of the minimum cost to obtain competitive victory is to find a seed set $S^{*}$, so that the influence of the set is greater than the influence of any other competitor and the total cost is the minimal. The formula is formatted as follows:

$$
S^{*}=\arg \min _{\sigma\left(S, C_{i}\right)>0} \operatorname{cost}(S)
$$

\section{Framework of the IMCLT-IM Algorithm for Competitive Influence Maximization}

In this paper, we propose a minimum cost influence maximization algorithm based on the competitive transition probability. In the model, we set a one-dimensional vector for each node, to store the temporal probability that the node chooses different competitors. In the process of spreading, we set the different weights for all competitors and activate the rest of the nodes according to a threshold rule we mentioned above. In each round of the seed node selection process, we select the nodes according to the cost-effectiveness of each node, and finally evaluate the influence based on the competitive transition probability.

Algorithm: IMCLT-IM

Input: $A$ : Adjacency matrix of network ;

$C$ : Set of competitor seed set;

$m$ : Number of competitors;

Output: $S$ : Set of seed nodes;

Begin:

1. Parameter initialization: the cost of each node $\operatorname{cost}(v)$, the probability selection node $P$;

2. while $\sigma\left(S, C_{i}\right)<0$

Select the node with the best cost-effective performance in the network to join the S set; Influence spreading based on the influence spreading model IMCLT, update $P$ according to formula (8);

Calculate the sum of the probabilities of all activated nodes belonging to a certain competitor.

4. End while

5. Output the Scoring matrix $S$ between the nodes.

End

\subsection{Detailed Algorithm}

Parameter initialization. Initialize probability matrix $P$ : in the initial state $(t=1)$, if the node $v$ is chosen as the seed node by competitor $i$, then the initial probability vector of the node is $P_{v}^{1}=(0,0, \ldots, 1,0,0)$, where the element on the $i$-th site is 1 and the others are 0 .

We use PageRank for the measurement of the cost of nodes in the network. PageRank is a 
metric that is used to sort web pages. As one of the most important applications, Google uses it to reflect the relevance and importance of web pages[33]. In this article we assume that the larger the PageRank value, the greater the cost of the node, and vice versa. The cost initialization formula for a node is computed as follows:

$$
\operatorname{cost}(v)=\operatorname{PageRank}(v)=(1-d)+d \sum_{u \in \Gamma(v)} \frac{\operatorname{PageRank}(u)}{\mathrm{C}(u)}
$$

where PageRank $(v)$ is the PageRank value of node $v$, and PageRank $(u)$ is the PageRank value of node $u, \Gamma(v)$ is the set of all nodes pointing to the $v$-node, and $\mathrm{C}(u)$ is the out-degree of node $u . d$ is the damping coefficient, which is the probability that the node will continue to propagate after reaching a node at any time. In this paper we set $d=0.85$.

The cost-effectiveness of the node is a measure of the performance of the candidate seed nodes in the network. According to the cost-effectiveness, the node with the highest costeffectiveness is first selected to join the set $S$. The cost-effectiveness formula $\operatorname{ppr}(v)$ for node $v$ is defined as follows:

$$
\operatorname{ppr}(\mathrm{v})=\frac{\sigma(S+v, C)-\sigma(S, C)}{\operatorname{cost}(v)}
$$

The numerator in the formula represents the difference in influence probability between the set $S$ before and after the new node $v$ joins the set, and the denominator represents the cost of the node $v$. From this we can see that the larger the difference, or the smaller the node cost, then the more cost-effective the node is. Thus, we choose the node with the highest costeffectiveness as the candidate seed node to join the set $S$.

The influence spreading is performed according to the spreading model IMCLT, and the node is activated if the formula (3) is satisfied.

We use $p_{i j}^{t}$ to indicate the probability that node $i$ chooses node $j$ at time $t$. After each iteration, the probability matrix of the activated node needs to be updated. The update rules are formulated as follows:

$$
p_{i j}^{t}=\frac{p_{i j}^{t-1}+\Delta p_{i j}^{t}}{\sum_{j=1}^{m} p_{i j}^{t-1}+\sum_{j=1}^{m} \Delta p_{i j}^{t-1}}
$$

where $\Delta p_{i j}^{t}=p_{i j}^{t-1} \times \frac{m-\operatorname{rank}(j)}{m}$ indicates the probability change of node $i$ selecting node $j$ at time $t$. $\operatorname{rank}(j)$ is the probability ranking of the $j$-th competitor among all competitors. If the ranking is lower, the spreading probability is greater, and vice versa. 


\section{Experimental Results and Analysis}

In this chapter, we leveraged two classical data sets from two different fields to carry out experiments: wiki-Vote (Wikipedia who-votes-on-whom network) and soc-Epinions1(Whotrusts-whom network of Epinions.com). For each of the datasets, we all used the maximum connected component as the benchmark data set. Table 1 describes the network topology characteristics of the two datasets. The number of nodes and the number of edges in the network are represented by $N$ and $M$, respectively. Kmax is the maximum degree. The average degree of a node is expressed by $K$. $S$ is the sparsity of network.

Table 1. Network topology characteristics of different data sets

\begin{tabular}{|c|c|c|c|c|c|}
\hline Networks & $\boldsymbol{N}$ & $\boldsymbol{M}$ & $\boldsymbol{K}$ & $\boldsymbol{K m a x}$ & $\boldsymbol{S}$ \\
\hline \hline wiki-Vote & 7115 & 103689 & 14.5733 & 457 & 0.002 \\
\hline soc-Epinions1 & 75879 & 508837 & 6.7051 & 3035 & 0.0000884 \\
\hline
\end{tabular}

In the experiment, we used the PageRank to calculate the cost of the node, we assumed the influence of the edge spreading probability $P=0.01$. We averaged for 10,000 times with the Monte Carlo method to calculate the Influences of set $S$ and the set $C$. We used the following three algorithms to compare with the IMCLT-IM algorithm: Max Degree, Random, and Greedy. In our experiments, we mainly tested and compared the runtime of various algorithms and the total cost of the target nodes. We assumed that competitors chose the seed node set $\mathrm{C}$ through a random algorithm. Since the nodes selected by the competitors may change in each simulation, we needed to average the results of simulations. We ran all algorithms ten times on different data sets, and then used the average of the ten operation time and the total cost as the final experimental results. Fig. 1 shows the total cost of a set of seed nodes selected by different algorithms on different data sets.
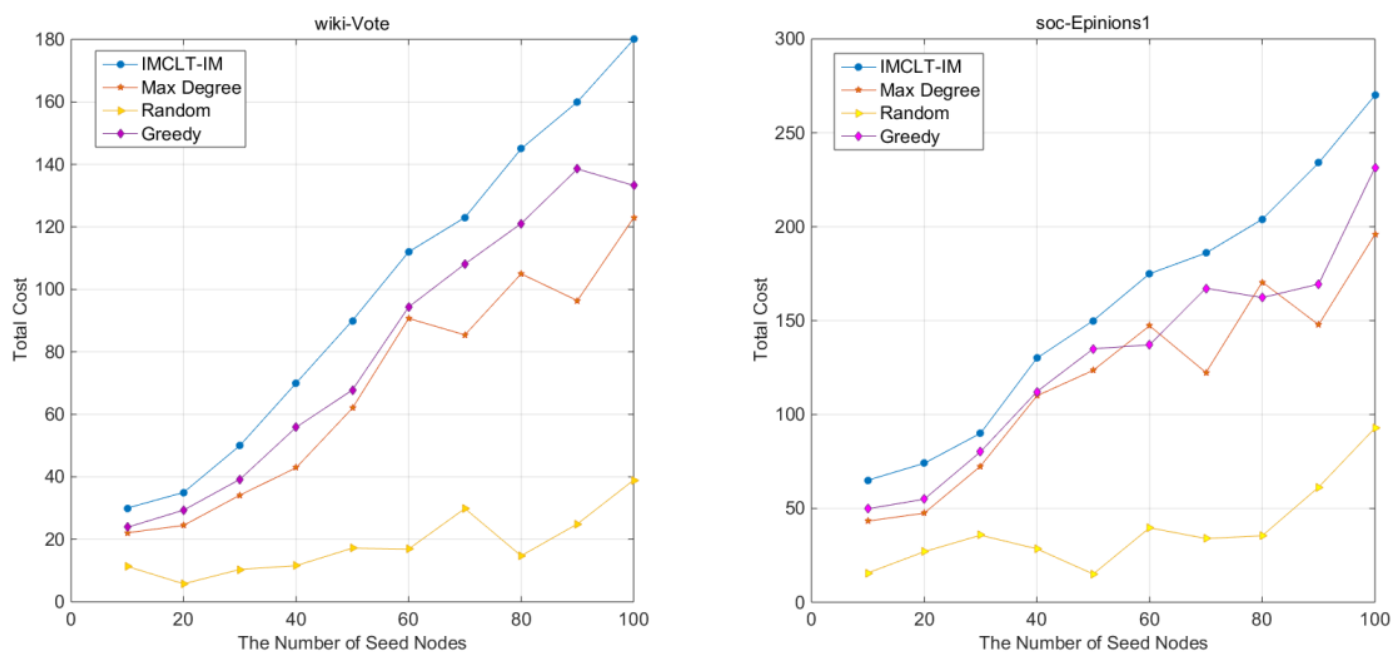

Fig. 1. Total cost of seed sets selected by different algorithms. The horizontal axis represents the total number of seeds in the seed set and the vertical axis represents the total cost of the seed set. 
From the figure we can see that as the number of seed nodes chosen by competitors increases, the total cost of the set of seed nodes $S$ determined by the algorithm was gradually increasing. Among them, the seed node set selected by the Random algorithm was unsurprisingly the worst, that is, it required the most cost to win over the competitors. The performance of the Max Degree algorithm and the Degree Adjust algorithm were similar, and both were greatly enhanced compared to the Random algorithm. The performance of the Costbased Degree Adjust algorithm was better than the Max Degree and Degree Adjust algorithm. Comparatively, the algorithm proposed in this paper has achieved the best results.

\section{Conclusion}

In the problem of influence maximizing of online social networks, each user may be influenced by different competitors to make product selection, but this effect is not fixed for a long time, so in this paper, we propose the IMCLT spreading model, a model that sets a proprietary weight to activate a neighbor node for all competitors. The state of node is either active or inactive, and can be only activated if the activation threshold is fulfilled. Based on the model, this paper designs a minimum cost influence maximization algorithm based on competitive transition probability. Each node has a probability of selecting different competitors at different times. Each time we choose the seed node on the basis of the cost-effectiveness of the node. Finally, the influence evaluation is based on the competitive transition probability. Experiments show that our proposed minimum cost competitive influence maximization algorithm based on the IMCLT propagation model can not only effectively reduce the time complexity of the algorithm, but also have excellent performance.

\section{Acknowledgement}

This research was supported in part by the National Natural Science Foundation of China under grants No.61602202 and 61702441, the Natural Science Foundation of Jiangsu Province under contract BK20160428, the Six talent peaks project in Jiangsu Province under contract XYDXX-034 and the key projects of Natural Science Foundation of universities in Jiangsu Province under contract 20KJA520008 and Innovation and entrepreneurship training program for college students in Jiangsu Province.

\section{References}

[1] R. M. Bond, C. J. Fariss, A. D. I. Kramer, C. Marlow, J. E. Settle, and J. H. Fowler, “A 61-millionperson experiment in social influence and political mobilization," Nature, vol. 489, no. 7415, pp. 295-298, 2012. Article (CrossRef Link)

[2] I. Gaye, G. Mendy, S. Ouya, and D. Seck, "Spanning graph for maximizing the influence spread in Social Networks," in Proc. of the 2015 IEEE/ACM International Conference on Advances in Social Networks Analysis and Mining, pp. 1389-1394, 2015. Article (CrossRef Link)

[3] Z. Lu, L. Fan, W. Wu, B. Thuraisingham, and K. Yang, "Efficient influence spread estimation for influence maximization under the linear threshold model," Computational Social Networks, vol. 1, no. 2, pp. 1-19, 2014. Article (CrossRef Link) 
[4] N. Barbieri, F. Bonchi, and G. Manco, "Topic-aware social influence spreading models," Knowledge and Information Systems, vol. 37, no. 3, pp. 555-584, 2013. Article (CrossRef Link)

[5] B. Bi, Y. Tian, Y. Sismanis, A. Balmin, and J. Cho, "Scalable topic-specific influence analysis on microblogs," in Proc. of the $7^{\text {th }}$ ACM International Conference on Web Search and Data Mining, pp. 513-522, 2014. Article (CrossRef Link)

[6] X. He and D. Kempe, "Stability of influence maximization," in Proc. of the $20^{\text {th }}$ ACM SIGKDD International Conference on Knowledge Discovery and Data Mining, pp. 1256-1265, 2014. Article (CrossRef Link)

[7] E. Cohen, D. Delling, T. Pajor, and R. F. Werneck, "Sketch-based influence maximization and computation: Scaling up with guarantees," in Proc. of the $23^{\text {rd }}$ ACM International Conference on Conference on Information and Knowledge Management, pp. 629-638, 2014. Article (CrossRef Link)

[8] F. Morone and H. A. Makse, "Influence maximization in complex networks through optimal percolation," Nature, pp. 65-68, 2015. Article (CrossRef Link)

[9] X. D. Wu, Y. Li, and L. Li, "Influence analysis of online social networks," Chinese Journal of Computers, vol. 37, no. 4, pp. 735-752, 2014. Article (CrossRef Link)

[10] Z. Zhu, "Discovering the influential users oriented to viral marketing based on online social networks," Physica A: Statistical Mechanics and its Applications, vol. 392, no. 16, pp. 3459-3469, 2013. Article (CrossRef Link)

[11] H. Wu, W. Liu, K. Yue, W. Huang, and K. Yang, "Maximizing the Spread of Competitive Influence in a Social Network Oriented to Viral Marketing," Web-Age Information Management, Springer International Publishing, pp. 516-519, 2015. Article (CrossRef Link)

[12] S. H. Lee, Y. Nah, and L. Choi, "Influenza surveillance and forecast with smartphone sensors," Computing, vol. 97, no. 3, pp. 237-259, 2015. Article (CrossRef Link)

[13] S. Chen and W. Tan, "Influence diffusion model in multiplex networks," Computers, Materials \& Continua, vol. 64, no. 1, pp. 345-358, 2020. Article (CrossRef Link)

[14] F. Fan, W. Wu, Z. Lu, W. Xu, and D. Z. Du, "Influence Diffusion, Community Detection, and Link Prediction in Social Network Analysis," Dynamics of Information Systems: Algorithmic Approaches. Springer, pp. 305-325, 2013. Article (CrossRef Link)

[15] Y. Dong, J. Tang, S. Wu, J. Tian, N. V. Chawla, J. Rao, and H. Cao, "Link prediction and recommendation across heterogeneous social networks," in Proc. of IEEE $12^{\text {th }}$ International Conference on Data Mining, pp. 181-190, 2012. Article (CrossRef Link)

[16] L. Muchnik, S. Aral, and S. J. Taylor, "Social influence bias: A randomized experiment," Science, vol. 341, no. 6146, pp. 647-651, 2013. Article (CrossRef Link)

[17] C. Cai, H. Xu, J. Wan, B. Zhou, and X. Xie, "An attention-based friend recommendation model in social network," Computers, Materials \& Continua, vol. 65, no. 3, pp. 2475-2488, 2020. Article (CrossRef Link)

[18] G. Zhang, J. Yang, Y. Zheng, Z. Luo, and J. Zhang, "Optimal Discriminative Feature and Dictionary Learning for Image Set Classification," Information Science, vol. 547, no. 8, pp. 498513, 2021. Article (CrossRef Link)

[19] G. Zhang, H. Sun, Y. Zheng, G. Xia, L. Feng, and Q. Sun, "Optimal Discriminative Projection for Sparse Representation-based Classification via Bilevel Optimization," IEEE Transactions on Circuits and Systems for Video Technology, vol. 30, no. 4, pp. 1065-1077, 2019. Article (CrossRef Link)

[20] E. Bakshy, D. Eckles, R. Yan, and I. Rosenn, "Social influence in social advertising: evidence from field experiments," in Proc. of the $13^{\text {th }}$ ACM Conference on Electronic Commerce, pp. 146161, 2012. Article (CrossRef Link) 
[21] L. Zhang, "The optimization analysis of the communication model of negative influence of the entrepreneur's social relationship change," Intelligent Automation \& Soft Computing, vol. 26, no. 3, pp. 577-583, 2020. Article (CrossRef Link)

[22] S. Bharathi, D. Kempe, and M. Salek, "Competitive influence maximization in social networks," in Proc. of International Workshop on Web and Internet Economics, pp. 306-311, 2007. Article (CrossRef Link)

[23] Y. Hua, B. Chen, Y. Yuan, G. Zhu, and J. Ma, "An influence maximization algorithm based on the mixed importance of nodes," Computers, Materials \& Continua, vol. 59, no. 2, pp. 517-531, 2019. Article (CrossRef Link)

[24] C. V. Pham, N. V. Nguyen, T. X. Le, H. X. Hoang, and M. T. Thai, "Competitive Influence Maximization on Online Social Networks: A Deterministic Modeling Approach," in Proc. of 2019 IEEE-RIVF International Conference on Computing and Communication Technologies (RIVF), vol. 9, no. 11, pp. 1-6, 2019. Article (CrossRef Link)

[25] C. V. Pham, H. V. Duong, and H. X. Hoang, "Competitive Influence Maximization within Time and Budget Constraints in Online Social Networks: An Algorithmic Approach," Applied Sciences, vol. 9, no. 11, 2019. Article (CrossRef Link)

[26] R. Yan, Y. Zhu, and D. Li, "Minimum cost seed set for threshold influence problem under competitive models," World Wide Web, pp. 2977-2996, 2019. Article (CrossRef Link)

[27] G. Gao, M. Xiao, J. Wu, H. Huang, and G. Chen, "Minimum Cost Seed Selection for Multiple Influences Diffusion in Communities," in Proc. of the $15^{\text {th }}$ International Conference on Mobile Ad Hoc and Sensor Systems (MASS), pp. 263-271, 2018. Article (CrossRef Link)

[28] F. Wang, W. Jiang, and X. Li, "Maximizing positive influence spread in online social networks via fluid dynamics," Future Generation Computer Systems, vol. 86, pp. 1491-1502, 2018. Article (CrossRef Link)

[29] M. Chen and L. Pan, "Least Cost Precision Marketing Based on User Profiles in Social Networks," in Proc. of the $3^{\text {rd }}$ International Conference on Security of Smart Cities, Industrial Control System and Communications (SSIC), pp. 1-8, 2018. Article (CrossRef Link)

[30] Y. Wang, W. Huang, L. Zong, T. Wang, and D. Q. Yang, "Influence maximization with limit cost in social network," Science China Information Sciences, vol. 56, no. 7, pp. 1-14, 2013. Article (CrossRef Link)

[31] Y. Zhu, D. Li, and Z. Zhang, "Minimum cost seed set for competitive social influence," in Proc. of IEEE INFOCOM 2016- The 35 th Annual IEEE International Conference on Computer Communications, pp. 1-9, 2016. Article (CrossRef Link)

[32] J. Yang and J. Liu, "Influence maximization-cost minimization in social networks based on a multiobjective discrete particle swarm optimization algorithm," IEEE Access, vol. 6, pp. 23202329, 2017. Article (CrossRef Link)

[33] P. Fernandez, "Google's PageRank and Beyond: The Science of Search Engine Rankings," The Mathematical Intelligencer, vol. 30, no. 1, pp. 68-69, 2008. Article (CrossRef Link) 


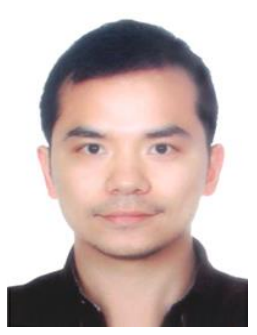

Bo-Lun Chen received the Ph.D. degree in computer science and technology from Nanjing University of Aeronautics and Astronautics, Jiangsu, China, in 2016. He is currently an Associate Professor with the Faculty of Computer and Software Engineering, Huaiyin Institute of Technology, Huaian, China. His research interests include complex network analysis and data mining.
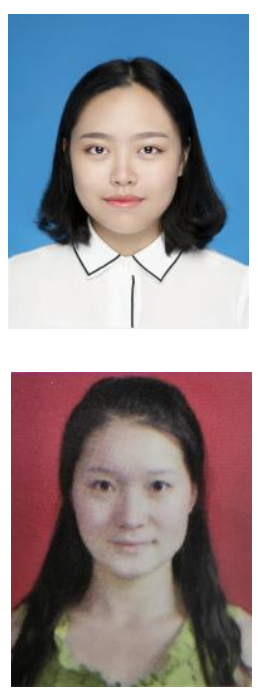

Min Ji received her M.S. degree from Yangzhou University. She is currently an Associate Professor with the Faculty of Computer and Software Engineering, Huaiyin Institute of Technology, Huaian, China. Her research interests include complex network analysis and deep learning.

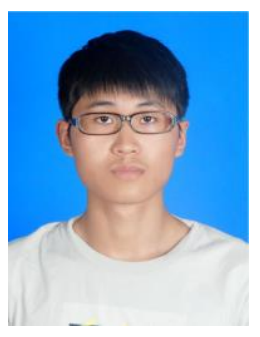

Ji-Wei Liu is currently pursuing a bachelor's degree at the Faculty of Computer and Software Engineering in Huaiyin Institute of Technology, Huaian, China. His current research interests include the deep learning and complex network analysis.

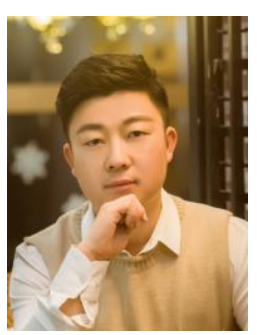

Yongtao Yu received the Ph.D. degree in computer science and technology from Xiamen University, Xiamen, China, in 2015. He is currently an Associate Professor with the Faculty of Computer and Software Engineering, Huaiyin Institute of Technology, Huaian, China. His research interests include intelligent transportation systems, computer vision, deep learning, and intelligent interpretation of 3-D point clouds and remotely sensed imagery.

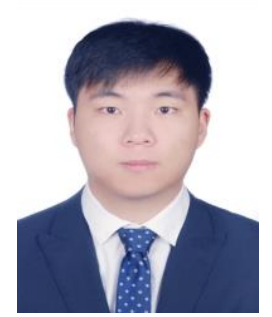

Yue Zhang received his bachelor's degree in Nanjing University, Jiangsu, China. His research interests include pattern recognition and artificial intelligence. 